International Mathematical Forum, Vol. 9, 2014, no. 5, 207 - 215

HIKARI Ltd, www.m-hikari.com

http://dx.doi.org/10.12988/imf.2014.37130

\title{
Miyachi's Theorems Associated with a Differential- Difference Operator on the Real Line
}

\author{
R. Daher and H. Lahlali \\ University of Hassan II, Faculty of Sciences Ain Chock \\ Laboratory T.A.G.S.D, Casablanca, Morocco
}

Copyright (c) 2014 R. Daher and H. Lahlali. This is an open access article distributed under the Creative Commons Attribution License, which permits unrestricted use, distribution, and reproduction in any medium, provided the original work is properly cited.

\begin{abstract}
We consider a singular differential operator $\triangle$ on the real line .we establish Miyachi's theorems for the generalized Fourier transform on $\mathbb{R}$ tied the Differential-difference operator .
\end{abstract}

Keywords: Differential-difference operator, generalized Fourier transform, Miyachi's theorems

\section{1- Introduction}

There are many theorems known which state that a function and its classical Fourier transform on $\mathbb{R}$ cannot both be sharply localized. That is, it is impossible for a non-zero function and its Fourier transform to be simultaneously small. Here a concept of the smallness had taken different interpretations in different contexts. Hardy[6], Cowling and Price[2], and Miyachi[9],for example, interpreted the smallness as sharp pointwise estimates or integrable decay of functions. Hardy's theorem[6] for the usual Fourier transform $\mathcal{F}$ on $\mathbb{R}$ asserts that $f$ and its Fourier $\widehat{f}=\mathcal{F}(f)$ can not both be very small. More precisely, let $a$ and $b$ be a positive constants and assume that $f$ is a measurable function on $\mathbb{R}$ such that $|f(x)| \leq C e^{-a x^{2}}$ a.e . and $|\widehat{f}(y)| \leq C e^{-b y^{2}}$ for some positive constant $C$. Then $f=0$ a.e. if $a b>\frac{1}{4}, f$ is a constant multiple of $e^{-a x^{2}}$ if $a b=\frac{1}{4}$, and there are infinitely many nonzero functions satisfying the assumptions if $a b<\frac{1}{4}$. Considerable attention has been devoted to 
discovering generalizations to new contexts for Hardy's theorem. In particular,Cowling and Price[2] have studied an $L^{p}$ version of Hardy's theorem which states that for $p, q \in[1, \infty]$, at least one of them is finite, if $\left\|e^{a x^{2}} f\right\|_{p}<\infty$ and $\left\|e^{b y^{2}} \widehat{f}\right\|_{q}<\infty$, then $f=0$ a.e. If $a b \geq \frac{1}{4}$. Another generalization of Hardy's theorem is given by Miyachi[9] where it is proved that, if $f$ is a measurable function on $\mathbb{R}$ such that

$$
e^{a x^{2}} f \in L^{1}(\mathbb{R})+L^{\infty}(\mathbb{R})
$$

and

$$
\int_{\mathbb{R}} \log ^{+} \frac{\left|\widehat{f}(\xi) e^{\frac{1}{4 a} \xi^{2}}\right|}{\lambda} d \xi<\infty
$$

for some positive constants $a$ and $\lambda$, then $f$ is a constant multiple of $e^{-a x^{2}}$. As a generalisation of these Euclidean uncertainty principles for $\mathcal{F}$, recently Mourou[10] have proved Hardy's theorem and Cowling Price's theorem for the Differential-Difference transform $F$, many authors have established the analogous of Cowling-Price's and Hardy's theorems in other various settings of harmonic analysis

(see for instance $[1,4,5,8,12,10])$. The purpose of this paper is a generalisation of Miyachi's theorem for $F$

The structure of this paper is as follows. In Section 2, we deal with harmonic analysis associated with the Differential-Difference operator.

The third section, is devoted to Miyachi's theorem for the DifferentialDifference transform. We establish that for all $p, q \in[1,+\infty]$ and $f$ is a mesurale function on $\mathbb{R}$ such that :

$$
e^{a x^{2}} f \in L^{1}(\mathbb{R})+L^{\infty}(\mathbb{R}) \quad \text { and } \quad \int \log ^{+} \frac{\left|F(f)(y) e^{b y^{2}}\right|}{\lambda} d y<\infty
$$

for some constants $a, b, \lambda>0$, If $a b>\frac{1}{4}$ then $f=0$ a.e ; If $a b=\frac{1}{4}$ then $f=C E_{a}$ with $|C| \leq \lambda$ and if $a b<\frac{1}{4}$ the function having form $f=C E_{t}$ then $F(f)(y)=C e^{-\frac{1}{4 t} y^{2}}$ and $f$ and $F(f)$ satisfying these conditions for all $t \in] a, \frac{1}{4 b}[$.

Throughout this paper, the letter $C$ indicates a positive constant that is not necessarily the same in each occurrence. 


\section{2- Differential-Difference transform}

In this paper; we consider the first-order singular differential - difference operator on $\mathbb{R}$

$$
\triangle f=\frac{d f}{d x}+\frac{A^{\prime}(x)}{A(x)}\left(\frac{f(x)-f(-x)}{2}\right)
$$

where: $\quad A(x)=|x|^{2 \alpha+1} B(x) \quad, \quad \alpha>-\frac{1}{2}$

$B$ being a positive $C^{\infty}$ even function on $\mathbb{R}$. We suppose in addition that :

(i) $A$ is increasing on $[0, \infty[$;

(ii) There exists a costant $\delta>0$. such that the function $e^{\delta x} \frac{B^{\prime}(x)}{B(x)}$ is bounded for large $x \in[0, \infty[$ together with its derivatives

The generalized Fourier transform related to $\triangle$ is defined for a suitable function $f$ on $\mathbb{R}$.by $\digamma f(\lambda)=\int_{\mathbb{R}} f(x) \Phi_{-i \lambda}(x) A(x) d x$. Where $\Phi_{-i \lambda}(x)$ is the solution of the differential -difference equation $\triangle u=-i \lambda u, u(0)=1$

The intention of this paper is to establish an analogue of Miyachi's theorems for the generalized Fourier transform $\digamma$.

\section{Preliminaries:}

In this section we recall some facts about harmonic analysis related to the differential-difference operator $\triangle$

We cite here ,as briefly as possible, only those properties actually required for the discussion .For more details we refer to[7]

If $\lambda \in \mathbb{C}$, it is know that the differential-difference equation $\triangle u=\lambda u$, $u(0)=1$, admits a unique $C^{\infty}$ solution on $\mathbb{R}$, denoted $\Phi_{\lambda}$ given by:

$$
\Phi_{\lambda}(x)=\left\{\begin{array}{lrl} 
& \varphi_{i \lambda}(x)+\frac{1}{\lambda} \frac{d}{d x} \varphi_{i \lambda}(x) & \text { if } \lambda \neq 0 \\
1 & \text { if } \lambda=0
\end{array}\right.
$$

$\Phi_{\lambda}(x)$ is entire in $\lambda$.

\section{Notation:}

For a Borel positive measure $\mu$ on $\mathbb{R}$, and $1 \leq p \leq \infty$, we write $L^{p}(\mathbb{R}, \mu)$ for the Lebesgue Space equiped with the norm \|\|$_{p, \mu}$ defined by

$\|f\|_{p, \mu}=\left(\int_{\mathbb{R}}|f(x)|^{p} d \mu(x)\right)^{\frac{1}{P}}$, if $p<\infty$, and $\|f\|_{\infty, \mu}=\operatorname{ess} \sup _{x \in \mathbb{R}}|f(x)|$

When $u=w(x) d x$, with $w$ a nonnegative function on $\mathbb{R}$, we replace the $\mu$ in the norms by $w$. 
The generalized Fourier transform of a function $f \in L^{1}(\mathbb{R}, A(x) d x)$ is defined by:

$$
\digamma f(\lambda)=\int_{\mathbb{R}} f(x) \times \Phi_{-i \lambda}(x) A(x) d x \quad, \quad \lambda \in \mathbb{R}
$$

a Plancherel type for the transform $\digamma$ is as follows.

Theorem 1 1. There is an even positive tempered measure $\sigma$ (and only one) on $\mathbb{R}$.such that for all $f \in L^{1} \cap L^{2}(\mathbb{R}, A(x) d x)$

$$
\int_{\mathbb{R}}|f(x)|^{2} A(x) d x=\int_{\mathbb{R}}|\digamma f(\lambda)|^{2} d \sigma(\lambda)
$$

2. The generalized Fourier transform $\digamma$ extends uniquely to a unitary isomorphism from : $L^{2}(\mathbb{R}, A(x) d x)$ onto $L^{2}(\mathbb{R}, \sigma)$. The inverse transform is given by:

$$
\digamma^{-1} g(x)=\int_{\mathbb{R}} g(\lambda) \times \Phi_{i \lambda}(x) d \sigma(\lambda)
$$

where the integral converges in $L^{2}(\mathbb{R}, A(x) d x)$.

The measure $\sigma$ is called . the spectral measure associated with the differentialdifference operator $\triangle$.under our assumptions on the fuction $A$, it is known $($ see $[7,11])$ that the spectral measure $\sigma$ takes the form

$$
d \sigma(\lambda)=\frac{d \lambda}{|c(|\lambda|)|^{2}} \quad, \quad \lambda \in \mathbb{R}
$$

where $c(s)$ is a cotinuous function on $] 0, \infty[$ such that :

$$
\begin{aligned}
& c(s)^{-1} \backsim k_{1} s^{\alpha+\frac{1}{2}} \quad \text { as } \quad s \longrightarrow \infty \\
& c(s)^{-1} \backsim k_{2} s^{\alpha+\frac{1}{2}} \quad \text { as } \quad s \longrightarrow 0
\end{aligned}
$$

for some $k_{1}, k_{2} \in \mathbb{C}$.

The generalized Gaussian kernel $E_{a}, a>0$,is defined by:

$$
E_{a}(x)=\int_{\mathbb{R}} e^{-\frac{\lambda^{2}}{4 a}} \varphi_{i \lambda}(x) d \sigma(\lambda), \quad x \in \mathbb{R} .
$$

From [3] we know that $E_{a}$ is a positive even function on $\mathbb{R}$, and belongs to the Schwartz space. Moreover,there are two positive constants $c_{1}$ and $c_{2}$ depending on $a$ such that:

$$
c_{1} e_{-a} \leq E_{a} \leq c_{2} e_{-a}
$$

where $e_{-a}(x)=e^{-a x^{2}}, x \in \mathbb{R}$ 


\section{3-Miyachi's theorems}

The proofs of this paper depends on the folowing lemmas

Lemma $1[10]$ Let $q \in[1, \infty], a>0$ and $\lambda=\xi+i \eta$ with $\xi, \eta \in \mathbb{R}$. Then there exists a positive constant $C$ such that

$$
\left\|e_{-a} \Phi_{-i \lambda}\right\|_{q, A} \leq C(1+|\eta|)^{\frac{2 \alpha+2}{q}} e^{\frac{\eta^{2}}{4 a}}
$$

$e_{-a}(x)=e^{-a x^{2}}$

Lemma 2 If $f(z)$ is an entiere function of $z \in \mathbb{C}$, if there exist constants $A, B>0$ and a positive integer $m$ such that

$$
|f(z)| \leq A(1+|z|)^{m} \exp \left(B(\Re e z)^{2}\right)
$$

and if

1 .

$$
\int_{-\infty}^{+\infty} \log ^{+} \frac{|f(t)|}{(1+t)^{m}} d t<\infty
$$

then $f(z)$ is a polynomial in $z$ of degree at most $m$

Corollary 1 If in Lemma2, the assumption (1) is replaced with

1.

$$
\int_{-\infty}^{+\infty} \log ^{+}|f(t)| d t<\infty
$$

then $f(z)$ is a constant.

Lemma 3 Let $p, q$ in $[1, \infty]$ and $f$ a measurable function on $\mathbb{R}$ such that:

$$
e^{a x^{2}} f \in L^{1}(\mathbb{R}, A)+L^{\infty}(\mathbb{R}, A)
$$

for some $a>0$.then for all $z \in \mathbb{C}$, the integral

$$
F(f)(\lambda)=\int_{\mathbb{R}} f(x) \Phi_{-i \lambda}(x) A(x) d x
$$

is well defined $F(f)(z)$ is entire and there exists $C>0$ such that for all $\zeta, \eta$ in $\mathbb{R}$

$$
|F(f)(\zeta+i \eta)| \leq C(1+|\eta|)^{2 \alpha+2} e^{\frac{\eta^{2}}{4 a}}
$$




\section{Proof:}

From analyticity theorem under the integral sign, we deduce that the function defined on $\mathbb{C}$ by $[13]$ is well defined and entire on $\mathbb{C}$

$$
\begin{aligned}
|F f(\lambda)| & =\left|\int_{\mathbb{R}} f(x) \Phi_{-i \lambda}(x) A(x) d x\right| \\
& =\left|\int_{\mathbb{R}} e^{a x^{2}} f(x) \times e^{-a x^{2}} \Phi_{-i \lambda}(x) A(x) d x\right| \\
& =\left|\int_{\mathbb{R}}\left(f_{1}(x)+f_{2}(x)\right) \times e^{-a x^{2}} \Phi_{-i \lambda}(x) A(x) d x\right| \quad\left(e^{a} f=f_{1}+f_{2} \in L^{1}(\mathbb{R}, A)+L^{\infty}(\mathbb{R}, A)\right) \\
& =\left|\int_{\mathbb{R}} f_{1}(x) \times e^{-a x^{2}} \Phi_{-i \lambda}(x) A(x) d x+\int_{\mathbb{R}} f_{2}(x) \times e^{-a x^{2}} \Phi_{-i \lambda}(x) A(x) d x\right| \\
& \leq\left\|f_{1}\right\|_{1, A} \times\left\|e^{-a} \Phi_{-i \lambda}\right\|_{\infty, A}+\left\|f_{2}\right\|_{\infty, A} \times\left\|e^{-a} \Phi_{-i \lambda}\right\|_{1, A} \quad \text { by Holder's inequality } \\
& \leq\left(C_{1}+C_{2}\right) \times C(1+|\operatorname{Im} \lambda|)^{2 \alpha+2} e^{\frac{(I m \lambda)^{2}}{4 a}} \quad \text { by Lemma } 1 \\
& \leq C^{\prime}(1+|\operatorname{Im} \lambda|)^{2 \alpha+2} e^{\frac{(I m \lambda)^{2}}{4 a}}
\end{aligned}
$$

Theorem $2 f$ is a mesurable function on $\mathbb{R}$ such that:

1.

$$
e^{a x^{2}} f \in L^{1}(\mathbb{R}, A)+L^{\infty}(\mathbb{R}, A)
$$

2.

$$
\int_{-\infty}^{+\infty} \log ^{+}\left|\frac{F f(\lambda) e^{b \lambda^{2}}}{C}\right| d \lambda<\infty
$$

for some constants $a, b, \lambda>0$ and $1 \leq p, q \leq+\infty$

(i) If $a b>\frac{1}{4}$ then $f=0$ almost everywhere

(ii) If $a b=\frac{1}{4}$ then $f=C E_{a}$ with $|C| \leq \lambda$

(iii) If $a b<\frac{1}{4}$ then the function having forme $f=C E_{t}$ then $F(f)=C e^{-\frac{1}{4 t} y^{2}}$

and $f$ and $F(f)$ satisfy (3.1) and (3.2) for all $t \in] a, \frac{1}{4 b}[$

\section{Proof:}

Let

$$
h(\lambda)=F f(\lambda) \times e^{\frac{\lambda^{2}}{4 a}}
$$




$$
\begin{aligned}
|h(\lambda)| & =|F f(\lambda)| \times\left|e^{\frac{\lambda^{2}}{4 a}}\right| \text { with } \lambda=\xi+i \eta \\
& =|F f(\lambda)| \times\left|e^{\frac{\xi^{2}}{4 a}}\right| \times\left|e^{-\frac{\eta^{2}}{4 a}}\right| \\
& \leq C e^{\frac{\eta^{2}}{4 a}} \times(1+|\eta|)^{2 \alpha+2} \times e^{\frac{\xi^{2}}{4 a}} \times e^{-\frac{\eta^{2}}{4 a}} \\
& \leq C \times(1+\eta)^{2 \alpha+2} \times e^{\frac{\xi^{2}}{4 a}}
\end{aligned}
$$

We will divide the proof into three cases.

(i) $a b>\frac{1}{4}(i) a b>\frac{1}{4}$.

$$
|h(\lambda)| \leq C(1+|\lambda|)^{2 \alpha+2} \times e^{\frac{(R e \lambda)^{2}}{4 a}}
$$

we note that

$$
\begin{aligned}
\int_{\mathbb{R}} \log ^{+}|h(y)| d y & =\int_{\mathbb{R}} \log ^{+}\left|e^{\frac{y^{2}}{4 a}} F(f)(y)\right| d y \\
& =\int_{\mathbb{R}} \log ^{+} \frac{\left|e^{b y^{2}} F(f)(y)\right|}{\lambda} \lambda e^{\left(\frac{1}{4 a}-b\right) y^{2}} d y \\
& \leq \int_{\mathbb{R}} \log ^{+} \frac{\left|e^{b y^{2}} F(f)(y)\right|}{\lambda} d y+\int_{\mathbb{R}} \lambda e^{\left(\frac{1}{4 a}-b\right) y^{2}} d y
\end{aligned}
$$

because $\log ^{+}(c d) \leq \log ^{+}(c)+d$ for all $c, d>0$, since $a b>\frac{1}{4},(3.2)$ implies that

$$
\int_{\mathbb{R}} \log ^{+}|h(y)| d y<+\infty
$$

then it follows from (3.3) and (3.4) that $h$ satisfies the assumptions in Lemma 2,and thus, $h$ is a constant and

$$
F(f)(y)=C e^{-\left(\frac{1}{4 a}\right) y^{2}}
$$

Since $a b>\frac{1}{4},(3.2)$ holds whenever $C=0$ and the injectivity of $F$ implies that $f=0$ almost everywhere.

(ii) $a b=\frac{1}{4}$, As in the previous case, it follows that $F(f)(y)=C e^{-\frac{y^{2}}{4 a}}$ then (3.2) holds whenever $|C| \leq \lambda$. 
then $f(x)=\int_{\mathbb{R}} F(f)(\lambda) \times \Phi_{i \lambda}(x) d \sigma(\lambda)=\int_{\mathbb{R}} C e^{-\frac{\lambda^{2}}{4 a}} \Phi_{i \lambda}(x) d \sigma(\lambda)=$ $C E_{a}(x)$

(iii) $a b<\frac{1}{4}$,if $f=C E_{t}$, then $F(f)(y)=C e^{-\frac{1}{4 t} y^{2}}$ and for all $\left.t \in\right] a, \frac{1}{4 b}[$

$e^{a x^{2}} f=e^{a x^{2}} C E_{t} \leq C e^{(a-t) x^{2}} \in L^{1}(\mathbb{R}) \subset L^{1}(\mathbb{R})+L^{\infty}(\mathbb{R})$ by $(2.1)$ and because $a-t<0$

$\int_{\mathbb{R}} \log ^{+} \frac{\left|F(f)(y) e^{b y^{2}}\right|}{\lambda} d y=\int \log ^{+} \frac{\left|C e^{\left(b-\frac{1}{4 t}\right) y^{2}}\right|}{\lambda} d y<\infty$ because $b-\frac{1}{4 t}<0$

then $f$ and $F(f)$ satisfy (3.1) and (3.2) for all $t \in] a, \frac{1}{4 b}[$

\section{References}

[1] N.B. Andersen, Hardy's theorem for the Jacobi transform. Hiroshima Math. J., 33 (2003), pp. 229-251

[2] M.G. Cowling, J.F. Price, Generalizations of Heisenberg's inequality. Lect. Notes Math., 992 (1983), pp. 443-449

[3]C. F. Dunkl, Integral kernels with reflection group invariance, Can. J. Math. 43 (1991), pp: 1213-1227.

[4] M. Ebata, On the Cowling-Price theorem for SU(1,1). Hiroshima Math. J., 31 (2001), pp. 409-423

[5] A. Fitouhi, N. Bettaibi, R.H. Bettaieb ,On Hardy's inequality for symmetric integral transforms and analogous. Appl. Math. Comput., 198 (2008), pp. $346-354$

[6] G.H. Hardy, A theorem concerning Fourier transforms J. Lond. Math. Soc., 8 (1933), pp. 227-231

[7] S. Kamefuchi and Y. Ohnuki, Quantum Field Theory and Parastatistics, University of Tokyo Press, Springer-Verlag, (1982).

[8] E.K. Narayanan, S.K. Ray, The heat kernel and Hardy's theorem on symmetric spaces of noncompact type. Proc. Indian Acad. Sci. Math. Sci., 112 (2) (2002), pp. 321-330

[9] A.Miyachi, A generalisation of theorem of Hardy, Harmonic Analysis Seminar held at Izunagaoka,Shizuoka-Ken, Japon 1997 , pp.593-606

[10]M. A. Mourou, Hardy and Cowling-Price Theorems associated with a Dunkl type Differentiak-Difference Operator on the real line,Conf. 02, pp. 56-62 (2008)

[11] M. Rosenblum, Generalized Hermite polynomials and the Bose like oscillator calculus,In Operator Theory : Advances and Applications, Birkh“auser Verlag, Vol. 73,(1994), pp. 369-396. 
[12] S. Thangavelu, Hardy's theorem for the Helgason Fourier transform on noncompact rank one symmetric spaces .Colloq. Math., 94 (2) (2002), pp. 263-280

[13] K. Trimèche, Cowling-Price and Hardy theorem's on Chébli-Trimèche hypergroups .Glob. J. Pure Appl. Math., 1 (3) (2005), pp. 286-305

Received: March 28, 2013 